\title{
Löcher im Kopf
}

Erhard Taverna

* Museum für Urgeschichte(n), Schädeloperationen in der Urgeschichte, bis 26. April 2009, Hofstrasse 15, Zug, Tel. 041 72828 80, www.museenzug.ch.
Die Geschichte der Trepanation oder Kraniotomie reicht bis in die Steinzeit zurück. Mittels Schaben, Bohren und Raspeln wurde der intakte Schädelknochen einer lebenden Person bis zur harten Hirnhaut geöffnet. Diese Schädeloperationen sind aus Europa, Asien, Amerika, Australien und von südpazifischen Inseln bekannt, wobei die ältesten Fälle aus Marokko und der Ukraine auf 12000 Jahre datiert werden. Oft sind gewaltsam zugefügte Begleitverletzungen nachweisbar, die auf eine Wundbehandlung zur Entfernung von Knochensplittern oder Blutungen unter der Schädelkalotte schliessen lassen. Wer sich über diese faszinierende Form frühgeschichtlicher Chirurgie informieren möchte, findet dazu im Museum für Urgeschichte(n) in Zug bis am 26. April 2009 eine informative Sonderausstellung über «Schädeloperationen in der Urgeschichte». ${ }^{*}$ Nach dem vom Naturhistorischen Museum Basel übernommenen Konzept, erklären die anschaulichen Exponate und Schautafeln die vielfältigen Operationstechniken mit Feuersteinklinge, Muschel, Meissel, Säge und Bohrer. Aus dem Grab eines Bingener Arztes um 100 n.Chr. stammt der bis in die Neuzeit verwendete Krontrepan, in diesem Fall ein bronzener Zylinder mit Sägezähnen und dem zugehörigen Trepanationsbogen. Schätzungen gehen davon aus, dass 70-80 Prozent der Patienten den Eingriff überlebten. Vermutlich wurden betäubende Mittel wie Alkohol oder Mohnsamen eingesetzt. Die Zuger Ausstellungsmacher zeigen als Zugabe eine grosse Auswahl von Pflanzen, darunter Bilsenkraut, Malve, Tollkirsche, Beinwell und Zaubernuss, die als anästhesierende oder desinfizierende Mittel in Frage kamen. Röntgenaufnahmen und MRI erlauben eine genaue Analyse der Bearbeitungsspuren und Knochenränder. Nachweisbare oder fehlende Heilungsprozesse ermöglichen eine Aussage über die Reaktion des Gewebes nach der Operation. Hiebverletzungen, Lochdefekte, wie sie bei Wasserleichen vorkommen, Missbildungen, Tumore oder Entzündungen durch Knochentuberkulose und Syphilis sind als weitere Ursachen abzugrenzen. Aus der Schweiz sind 22 Trepanationsfunde aus neolithischen (6000-2200 v. Chr.), bronzezeitlichen (2200-800 v.) und eisenzeitlichen (80015 v. Chr.) Epochen, aus der Römerzeit (15 v. Chr.400 n. Chr.) und dem Frühmittelalter (400- ca. 1000 n. Chr.) bekannt. Zur Ausstellungsdokumentation gehört eine unveröffentlichte medizinische Dissertation von 1976 über die Ur- und frühgeschichtlichen Schädeltrepanationen der Schweiz.

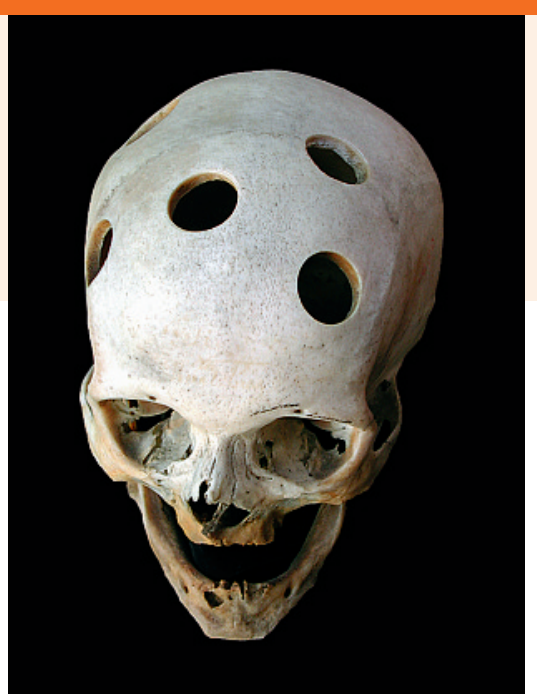

Die bearbeitete und ergänzte Fassung bietet mit zahlreichen Fotografien eine Übersicht als Grundlage für ein weiterführendes Forschungsprojekt. Wie im übrigen Europa finden sich die meisten Löcher auf der linken Stirn- und Scheitelbeinregion als ungefährlichster Zugang. Über die Operationsindikationen kann meistens nur spekuliert werden. Aus pathologischen Befunden am postkranialen Skelett, wie Karies, Frakturen und Osteomyelitis, schliessen einzelne Autoren auf eine Schmerztherapie. Epilepsie mag so behandelt worden sein, denn herausgetrennte Schädelfragmente wurden lange Zeit als Amulett gegen dieses Anfallsleiden getragen.

Oder waren es doch ausschliesslich magischreligiöse Rituale? In Melanesien wurden die kindlichen Fontanellen zur Verlängerung des Lebens trepaniert, in Ostafrika, bis vor ungefähr 40 Jahren, präventiv gegen Kopfweh und Wahnsinn geschabt. Die pseudowissenschaftliche Interpretation der Trepanation treibt bis in die Gegenwart skurrile Blüten. Eine Arbeit aus der Abteilung Allgemeine Psychologie der Universität Zürich von 2003 beschreibt eine sektenartige Gruppe trepanierter Personen, die sich 1998 in Amsterdam zu einem Weltkongress versammelten. Unter Anleitung des Gurus dieser International Advocacy Group wird die Auto-(Selbst-)Trepanation mit einem Elektrobohrer zur Erlangung eines erweiterten, sprich erhöhten Bewusstseins propagiert.

Ganz so weit wie diese «Selbsttrepanierer» mit dem Akkubohrer vor dem Badezimmerspiegel will die Ausstellung in Zug nicht gehen, auch wenn in anderen Museen zum gleichen Thema, an Aktionstagen Filmausschnitte zu dieser makabren Anwendung gezeigt wurden und die Besucher am modernen OP-Tisch Trepanationswerkzeuge selber ausprobieren konnten. Dafür gibt es im Museum für Urgeschichte mit dem schönen Ausblick auf den nahen Zuger See Vorträge und Führungen, ein Kinderferienprogramm, Instrumente zum Anfassen und im Rahmen der Erlebnisarchäologie ein Speerschleuder- und Bogenbauseminar; aber nur in Begleitung Erwachsener! 